\title{
A Multi-COMMOdity DisCRETE/CONTINUUM MODEL FOR A TRAFFIC EQUILIBRIUM SYSTEM
}

\author{
Y.C. DU \\ Key Laboratory of Road and Traffic Engineering of the Ministry of Education, \\ Tongji University, 1239 Siping Road, Shanghai 200092, P.R. China. \\ E-mail: ycdu@mail.tongji.edu.cn

\begin{abstract}
S.C. WONG
Department of Civil Engineering, The University of Hong Kong, Pokfulam Road, Hong Kong, P.R. China. E-mail: hhecwsc@hkucc.hku.hk
\end{abstract}

\section{L.J. SUN}

Key Laboratory of Road and Traffic Engineering of the Ministry of Education, Tongji University, 1239 Siping Road, Shanghai 200092, P.R. China.

E-mail: ljsun@mail.tongji.edu.cn

\begin{abstract}
We consider a city with several highly compact central business districts (CBDs). The commuters' origins are continuously dispersed. The travel demand to each CBD, which is considered to be a distinct commodity of traffic movements, is dependent on the total travel cost to that CBD. The transportation system is divided into two layers: major freeways and a dense network of surface streets. Whereas the major freeway network is modeled according to the conventional discrete network approach, the dense surface streets are approximated as a continuum. Travelers to each CBD can either travel within the continuum (surface streets) and then transfer to the discrete network (freeways) at an interchange (ramp) before moving to the CBD on the discrete network, or they can travel directly to the CBD within the continuum. Specific travel cost-flow relationships for the two layers of transportation facilities are considered. We develop a traffic equilibrium model for this discrete/continuum transportation system in which, for each origin-destination pair, no traveler can reduce his or her individual travel cost by unilaterally changing routes. The problem is formulated as a simultaneous optimization program with two sub-problems. One sub-problem is a traffic
\end{abstract}


assignment problem from the interchanges to the CBD in the discrete network, and the other is a traffic assignment problem within a continuum system with multiple centers (i.e., the interchange points and the CBDs). A Newtonian algorithm based on sensitivity analyses of the two sub-problems is proposed to solve the resultant simultaneous optimization program. A numerical example is given to demonstrate the effectiveness of the proposed method.

Keywords: Discrete system, continuum system, multi-commodity, traffic equilibrium, elastic demand

\section{INTRODUCTION}

The rapid development of the transportation network equilibrium problem in recent decades has led to a large body of literature devoted to the theory of equilibrium prediction and to computer algorithms for the determination of flows. In the literature, two general approaches are used to deal with network equilibrium problems. The first is discrete modeling of transportation networks, in which the zones are identified as nodes in space and the roads are treated as links between these nodes. The other approach is continuum modeling of network problems. The major assumption of this latter form of modeling is that the differences between adjacent areas within a network are relatively small when compared to the variations over the entire network, and hence the characteristics of the network can be represented by smooth mathematical functions (Vaughan, 1987).

Discrete modeling has been widely adopted for equilibrium prediction of transportation networks. Fernandez and Friesz (1983) and Boyce (1984) reviewed the different discrete approaches and methods that were proposed to deal with the equilibrium problem in a transportation network. Boyce and Daskin (1997) published a more recent review. Although the network equilibrium travel and route choice problem has been vigorously formulated and analyzed in considerable depth with the discrete approach, there is room for further improvement. First, although the algorithms developed to solve network equilibrium problems are good for small-scale networks, they are not generally efficient for dense transportation networks that involve many nodes and links and many possible routes between the origin-destination (O-D) pairs. Enormous computing time is consumed in the evaluation of the feasible flow at the equilibrium point for dense networks. Moreover, the computer solution for a very large network may give the flow pattern in such detail that it obscures 
some of the qualitative interpretation of the results. It is also difficult to determine from computer solutions the manner in which the flow varies as one changes the properties of the network except by an exhaustive evaluation of the flows for a wide range of possible networks. Moreover, the discrete approach has an inherent drawback whereby the demand is assumed to be concentrated at nodes called centroids and the traffic enters the discrete network by means of a few pre-specified centroid connectors. This assumption is not realistic in modeling traffic demand, which is actually distributed over the entire study area.

An alternative approach to the network equilibrium problem is to use continuum modeling for the network representation. The continuum approach usually involves only a small number of parameters that represent the network characteristics. The interrelation among these parameters can be determined with the help of a computer. Because the number of unknowns is lower than that for discrete modeling for a dense network, the computational effort is greatly reduced. In the best case, a simple analytical solution can be derived directly from the model. This information about the influence of various parameters is very useful in transportation planning, especially in the global sense. The continuum modeling of transportation systems can be broadly classified into two categories: specific city configuration approaches (Lam and Newell, 1967; Zitron, 1974; D’Este, 1987; Wong, 1994) and general city configuration approaches (Beckmann, 1952; Wardrop, 1971; Williams and Ortuzar, 1976; Puu, 1977; Buckley, 1979; Dafermos, 1980).

Recently, Taguchi and Iri (1982) proposed a promising numerical procedure to solve the problem of a continuum transportation system for a general city configuration in which the finite-element method (Zienkiewicz and Taylor, 1989) was used to solve three continuum problems: the maximum flow problem, the shortest route problem, and the minimum-cost flow problem. For user equilibrium problems, Sasaki et al. (1990) provided a dual-based formulation in which the user equilibrium problem in a continuum system was solved by minimizing an objective function that was subject to a set of constraints. The finite-element method was also used to determine the cost potential in the city, and the flow intensity was then deduced from the potential function. To improve the numerical stability of this solution, a more robust algorithm was developed on the basis of the mixed finite-element formulation (Wong et al., 1998); this algorithm was also applied to solve the continuum modeling of a multi-commodity traffic assignment with fixed and variable demands (Wong, 1998) and a combined distribution and assignment problem (Wong and Sun, 2001; Wong et al., 2004). 
For the problems of competitive facilities, Wong and Yang (1999) developed a continuum formulation for the determination of market areas and significantly extended it to incorporate the demand elasticity of customers over the space and market externality of each competitive facility (Yang and Wong, 2000). This extension added realism because congestion externalities and demand elasticity exist in practice. More recently, the continuum model has also been applied to solve multiclass equilibrium problems (Ho et al., 2003, 2004, 2006, 2007), cordon-based congestion-pricing problems (Ho et al., 2005, 2013), dynamic paradigm problems (Jiang et al., 2011; Du et al., 2013; Tao et al., 2014), environmental problems (Yin et al., 2012, 2013), airport problems (Loo et al., 2005), and housing problems (Ho and Wong, 2005, 2007).

Although the traffic equilibrium problem has been vigorously formulated and analyzed in considerable depth for both the discrete and continuum approaches, these approaches have different advantages and shortcomings, and it is promising to integrate them to form a combined model. D’Este (1987) studied discrete/continuum modeling by considering the problem of flow-dependent trip assignment in a city with a small number of radial major roads. He derived a system of differential equations for the spatial pattern of trip assignment in a model city with a continuous distribution of home locations and a ring-radial road network. Wong (1994) reformulated the problem as a minimization problem of an objective function that was subject to a set of constraints and solved by means of power series expansion. For a general city configuration, Yang et al. (1994) developed a dual-based formulation and a finite-element solution for a discrete/continuum network problem. In their approach, the solution algorithm is applicable to a special case in which only one feasible path exists from each interchange to the CBD. This restricts the solution's applicability to a more general discrete/continuum transportation system configuration. Wong et al. (2003) proposed an improved method in which the problem was reformulated as a simultaneous optimization model and solved it by means of a fixed-point algorithm based on sensitivity analyses of both the discrete and continuum systems. In this paper, we extend that method to the case of a multi-commodity network with variable demand, which has substantially improved the modeling capability of this discrete/continuum approach and made it applicable to a more general transportation system with multiple origins and destinations.

This paper is organized as follows. In Section 2, we introduce the modeled city with our assumptions, definitions, and notation. Section 3 describes the problem formulation, and 
Section 4 presents the solution algorithm for the resultant problem. Section 5 uses a numerical example to demonstrate the effectiveness of the proposed method.

\section{NOTATION AND ASSUMPTIONS}

\subsection{The modeled city}

We consider a city with several highly compact central business districts (CBDs), as shown in Figure 1, whose transportation system consists of two sub-systems: a discrete system for the freeway network and a continuum system for the dense network of surface streets. The discrete and continuum systems interact at the interchange locations (ramps), at which the travelers are collected to board the freeway network, and at the locations at which the travelers enter the CBDs. The commuters' origins are continuously dispersed. We assume that the travel demand to each CBD is dependent on the total travel cost to that CBD. There are two possible manners in which travelers can move to the CBDs. They can either travel within the continuum (surface streets) and then exchange to the discrete network (freeways) at an interchange (ramp) before moving to the CBD on the discrete network, or they can travel directly to the CBD within the continuum. This approach provides a more realistic model for the transportation system, in which the travel demand is continuously distributed over the city rather than concentrated at arbitrarily chosen point sources (centroids) and in which most travelers, except those who are close to the CBDs, tend to find a nearby interchange to transfer onto the discrete freeway network to take advantage of high-speed movement.

[Figure 1 to be inserted here]

\subsection{Continuum system}

The dense network of surface roads is approximated as a continuum, as shown in Figure 1c (Sasaki et al., 1990). There are $M$ CBDs and $N$ interchanges. These CBDs and interchanges are sufficiently compact for the continuum domain of the city. Denote the continuum region of the city as $\Omega$, the boundary of the city as $\Gamma$, and the location of each $\operatorname{CBD}$ as $\mathrm{O}_{m}, m=1,2$, ..., M. Note that a CBD can also act as an interchange for the other CBDs. Therefore, we 
define for each CBD $m$ the set of feasible interchanges (including the CBD $m$ itself) as $I_{m}$ and the location of each of these interchanges as $\mathrm{I}_{m n}, n=1,2, \ldots, N_{m}$, where $N_{m}$ is the number of feasible interchanges to CBD $m$. Note that $\mathrm{I}_{m 1}$ denotes the location of CBD $m$ (i.e., $\mathrm{I}_{m 1}=\mathrm{O}_{m}$ ) and that $N_{m} \leq M+N$. Each of these interchanges is of finite size and is enclosed by a clockwise boundary segment, $\Gamma_{m n}^{\mathrm{c}}, n=1,2, \ldots, N_{m}, m=1,2, \ldots, M$.

As in conventional traffic assignment, the travel demand to each CBD (i.e., destination) is considered as a distinct commodity and is assumed to be continuously distributed over city $\Omega$. This demand is represented by a nonnegative, heterogeneous density function $q_{m}(x, y)$, where $q_{m}$ is the total demand per unit area from the home location $(x, y) \in \Omega$. To consider the elasticity of travel demand, $q_{m}(x, y)$ is assumed to be a function of the minimum travel cost

$$
q_{m}(x, y)=D_{m}\left(u_{m}(x, y), x, y\right),
$$

where $u_{m}(x, y)$ and $q_{m}(x, y) \mathrm{d} x \mathrm{~d} y$ are the minimum travel cost and the travel demand, respectively, that are generated from the location $(x, y) \in \Omega$ to travel to CBD $m$. The function $D_{m}(., x, y)$ is assumed to decrease monotonically to reflect the elastic nature of travel demand with respect to the total cost, and its inverse function exists.

The local travel cost in the continuum domain of the city is assumed to depend on the local flow intensity and road configuration, but not on the direction (the isotopic case),

$$
c(x, y, \mathbf{f})=\alpha(x, y)+\beta(x, y)\left(\sum_{m=1}^{M}\left|\mathbf{f}_{m}(x, y)\right|\right)^{\gamma(x, y)},
$$

where $c(x, y, \mathbf{f})$ is the cost per unit distance of travel at coordinate $(x, y) \in \Omega, \alpha(x, y), \beta(x, y)$ and $\gamma(x, y)$ are strictly positive scalar functions of the cost-flow relationship that reflect the local characteristics of the streets, $\alpha(x, y)$ is the free-flow transportation cost per unit distance at coordinate $(x, y) \in \Omega, \beta(x, y)$ is the sensitivity parameter that represents the congestion effect at coordinate $(x, y) \in \Omega, \gamma(x, y)$ is a positive conversion factor that transforms the actual cost component to the perceived cost component that is experienced by class $\mathrm{m}$ users, $\mathbf{f}=\left(\mathbf{f}_{m}, m=1,2, \ldots, M\right), \mathbf{f}_{m}(x, y)=\left(f_{x m}(x, y), f_{y m}(x, y)\right)$ is a vector that represents the flow state of commodity $m$ in the city, $f_{x m}(x, y)$ and $f_{y m}(x, y)$ are the flow flux of commodity $m$ in the $x$ and $y$ directions, respectively, and 


$$
\left|\mathbf{f}_{m}(x, y)\right|=\sqrt{f_{x m}(x, y)^{2}+f_{y m}(x, y)^{2}},
$$

is the norm of the flow vector at $(x, y)$. This cost-flow relationship is generalized from the linear model by Wong (1998). A practical calibration process for the unit transportation cost function adopted in the continuum model was introduced by Wong and Wong (2015).

Inside the continuum domain of city $\Omega$, the flow vector and travel demand of each commodity must satisfy the flow conservation condition as

$$
\nabla \cdot \mathbf{f}_{m}(x, y)+q_{m}(x, y)=0, \quad \forall(x, y) \in \Omega, m=1,2, \ldots, M .
$$

Assuming that no traffic flow crosses the boundary of the city, we have $\mathbf{f}_{m}=\mathbf{0}, \forall(x, y) \in \Gamma \cup \Gamma_{m, N_{m}+1}^{\mathrm{c}} \cup \Gamma_{m, N_{m}+2}^{\mathrm{c}} \cdots \cup \Gamma_{m, M+N}^{\mathrm{c}}$, where $\Gamma_{m, N_{m}+1}^{\mathrm{C}}, \ldots, \Gamma_{m, M+N}^{\mathrm{C}}$ are the boundaries of the infeasible interchanges for commodity $m$. It is, however, not too difficult to extend the model to deal with the case that $\mathbf{f}_{m} \cdot \mathbf{n}=g_{m}(x, y)$ on boundary $\Gamma$, where $\mathbf{n}$ is the normal vector on the boundary and $g_{m}$ is a function representing the given demand distribution entering or leaving the city through the boundary. Denote

$$
\tilde{\Gamma}_{m}=\Gamma \cup \Gamma_{m, N_{m}+1}^{\mathrm{c}} \cup \Gamma_{m, N_{m}+2}^{\mathrm{c}} \cdots \cup \Gamma_{m, M+N}^{\mathrm{c}} \cdot
$$

as the combined boundary for commodity $m$, at which $\mathbf{f}_{m}=\mathbf{0}, \forall(x, y) \in \widetilde{\Gamma}_{m}, m=1,2, \ldots, M$.

Denote $\Omega_{m n}$ as the catchment area of interchange $n$ for commodity $m$. The travel demand attracted to this interchange can be expressed as

$$
Q_{m n}=\iint_{\Omega_{m n}} q_{m}(x, y) \mathrm{d} \Omega, \quad n=1,2, \ldots, N_{m}, m=1,2, \ldots, M,
$$

where $Q_{m 1}$ is the demand that travels directly to the CBD $m$ via the continuum only. Denote $\mathbf{Q}=\left(Q_{m n}, n=1,2, \ldots, N_{m}, m=1,2, \ldots, M\right)$. From the flow conservation principle, at each interchange, we have:

$$
\oint_{\Gamma_{m n}^{c}} \mathbf{f}_{m} \cdot \mathbf{n} \mathrm{d} \Gamma+Q_{m n}=0, \quad n=1,2, \ldots, N_{m}, m=1,2, \ldots, M .
$$

\subsection{Discrete system}

The discrete freeway network is described by a graph $G(V, A)$ in which $V$ is the set of discrete nodes including the CBDs, the interchanges, and the intermediate nodes and $A$ is the set of 
links, as shown in Figure 1b. Denote $v_{a}$ as the traffic flow on link $a$ of the freeway network and the set of link flows as $\mathbf{v}=\left(v_{a}, a \in A\right)$. The travel cost function on link $a$ is described by

$$
\bar{c}_{a}\left(v_{a}\right)=\bar{\alpha}_{a}+\bar{\beta}_{a} v_{a}^{\bar{\gamma}} .
$$

where $\bar{\alpha}_{a}, \bar{\beta}_{a}$, and $\bar{\gamma}$ are strictly positive coefficients. Let $P_{m n}$ be the set of paths from interchange $n$ to CBD $m$ and the traffic flow on path $p$ be $h_{m n p}, p \in P_{m n}, n=2, \ldots, N_{m}, m=$ $1,2, \ldots, M$. The traffic demand $Q_{m n}$ that is attracted to interchange $n$ heading to CBD $m$, as determined from equation (7), will form the O-D demand in the discrete network, and $\mathbf{Q}$ represents the O-D matrix for the network. Denote $\delta_{m n p a}$ as the link path incidence value, where $\delta_{\text {mnpa }}=1$ when path $p$ from interchange $n$ heading to $\operatorname{CBD} m$ uses link $a$, and $\delta_{\text {mnpa }}=0$ otherwise. We have

$$
v_{a}=\sum_{m=1}^{M} \sum_{n=2}^{N} \sum_{p \in P_{m n}} \delta_{m n p a} h_{m n p} .
$$

Further denote $U_{m n}$ as the equilibrium travel cost from interchange $n$ to CBD $m$ via the freeway network. Naturally, we have $U_{m 1}=0$.

\section{FORMULATION OF THE PROBLEM}

\subsection{Continuum system}

The user equilibrium problem for the continuum system can be formulated as the following mathematical program (P1).

$$
\begin{aligned}
\underset{\mathbf{f}}{\operatorname{Minimize}} Z(\mathbf{f})= & \sum_{m=1}^{M} \sum_{n=1}^{N} U_{m n} Q_{m n} \\
& +\iint_{\Omega}\left\{\alpha \sum_{m=1}^{M}|\mathbf{f}|+\frac{\beta}{\gamma+1}\left(\sum_{m=1}^{M}|\mathbf{f}|\right)^{\gamma+1}\right. \\
& \left.\quad-\iint_{\Omega} \sum_{m=1}^{M} \int_{0}^{q_{m}} D_{m}^{-1}(\omega) \mathrm{d} \omega\right\} \mathrm{d} \Omega,
\end{aligned}
$$

subject to

$$
\begin{gathered}
\nabla \cdot \mathbf{f}_{m}+q_{m}=0, \quad \forall(x, y) \in \Omega, m=1,2, \ldots, M, \\
\mathbf{f}_{m}=\mathbf{0}, \quad \forall(x, y) \in \widetilde{\Gamma}_{m}, m=1,2, \ldots, M, \\
\oint_{\Gamma_{m n}^{c}} \mathbf{f}_{m} \cdot \mathbf{n} \mathrm{d} \Gamma+Q_{m n}=0, \quad n=1,2, \ldots, N_{m}, m=1,2, \ldots, M .
\end{gathered}
$$


where boundary $\widetilde{\Gamma}_{m}$ for commodity $m$ is defined in equation (5). Consider the following Lagrangian,

$$
\begin{aligned}
\Pi= & \sum_{m=1}^{M} \sum_{n=1}^{N} U_{m n} Q_{m n} \\
& +\iint_{\Omega}\left\{\alpha \sum_{m=1}^{M}|\mathbf{f}|+\frac{\beta}{\gamma+1}\left(\sum_{m=1}^{M}|\mathbf{f}|\right)\right. \\
& \left.\quad-\iint_{\Omega} \sum_{m=1}^{M} \int_{0}^{q_{m}} D_{m}^{-1}(\omega) \mathrm{d} \omega+\sum_{m=1}^{M} u_{m}\left(\nabla \cdot \mathbf{f}_{m}+q_{m}\right)\right\} \mathrm{d} \Omega \\
& +\sum_{m=1}^{M} \int_{\tilde{\Gamma}_{m}} \mathbf{w}_{m} \cdot \mathbf{f}_{m} \mathrm{~d} \Gamma+\sum_{m=1}^{M} \sum_{n=1}^{N} \pi_{m n}\left(\oint_{\Gamma_{m n}^{c}} \mathbf{f}_{m} \cdot \mathbf{n} \mathrm{d} \Gamma+Q_{m n}\right),
\end{aligned}
$$

where $u_{m}(x, y), \mathbf{w}_{m}(x, y)=\left(w_{x m}(x, y), w_{y m}(x, y)\right)$, and $\pi_{m n}$ are the Lagrange multipliers associated with constraints (10b), (10c), and (10d), respectively. From the variational principle, let $\delta \mathbf{f}_{m}=\left(\delta f_{x m}, \delta f_{y m}\right), m=1,2, \ldots, M$, be arbitrary functions that vanish on boundary $\tilde{\Gamma}_{m}$, i.e., $\delta \mathbf{f}_{m}=\mathbf{0}, \forall(x, y) \in \widetilde{\Gamma}_{m}$. We can easily show that

$$
\begin{aligned}
& \delta \Pi=\iint_{\Omega}\left\{\sum_{m=1}^{M}\left(\alpha+\beta\left(\sum_{r=1}^{M}\left|\mathbf{f}_{r}\right|\right)^{\gamma}\right) \frac{1}{\left|\mathbf{f}_{m}\right|} \mathbf{f}_{m} \cdot \delta \mathbf{f}_{m}-\sum_{m=1}^{M} D_{m}{ }^{-1}\left(q_{m}\right) \delta q_{m}\right. \\
&\left.\quad+\sum_{m=1}^{M} u_{m}\left(\nabla \cdot \delta \mathbf{f}_{m}+\delta q_{m}\right)+\sum_{m=1}^{M} \delta u_{m}\left(\nabla \cdot \mathbf{f}_{m}+q_{m}\right)\right\} \mathrm{d} \Omega \\
&+\sum_{m=1}^{M} \sum_{n=1}^{N} U_{m n} \delta Q_{m n}+\sum_{m=1}^{M} \int_{\tilde{\Gamma}_{m}}\left(\mathbf{w}_{m} \cdot \delta \mathbf{f}_{m}+\delta \mathbf{w}_{m} \cdot \mathbf{f}_{m}\right) \mathrm{d} \Gamma \\
&+\sum_{m=1}^{M} \sum_{n=1}^{N} \delta \pi_{m n}\left(\oint_{\Gamma_{m n}^{\mathrm{c}}} \mathbf{f}_{m} \cdot \mathbf{n} \mathrm{d} \Gamma+Q_{m n}\right) \\
&+\sum_{m=1}^{M} \sum_{n=1}^{N} \pi_{m n}\left(\oint_{\Gamma_{m n}^{\mathrm{c}}} \delta \mathbf{f}_{m} \cdot \mathbf{n} \mathrm{d} \Gamma+\delta Q_{m n}\right) .
\end{aligned}
$$

By substituting $\nabla \cdot\left(u_{m} \delta \mathbf{f}_{m}\right)=\nabla u_{m} \cdot \delta \mathbf{f}_{m}+u_{m} \nabla \cdot \delta \mathbf{f}_{m}$, we have

$$
\begin{aligned}
& \delta \Pi=\iint_{\Omega}\left\{\sum_{m=1}^{M}\left(\alpha+\beta\left(\sum_{r=1}^{M}\left|\mathbf{f}_{r}\right|\right)^{\gamma}\right) \frac{1}{\left|\mathbf{f}_{m}\right|} \mathbf{f}_{m} \cdot \delta \mathbf{f}_{m}-\sum_{m=1}^{M} D_{m}{ }^{-1}\left(q_{m}\right) \delta q_{m}\right. \\
& \quad+\sum_{m=1}^{M} \nabla \cdot\left(u_{m} \delta \mathbf{f}_{m}\right)-\sum_{m=1}^{M} \nabla u_{m} \cdot \delta \mathbf{f}_{m}+\sum_{m=1}^{M} u_{m} \delta q_{m} \\
&\left.\quad+\sum_{m=1}^{M} \delta u_{m}\left(\nabla \cdot \mathbf{f}_{m}+q_{m}\right)\right\} \mathrm{d} \Omega \\
&+\sum_{m=1}^{M} \sum_{n=1}^{N} U_{m n} \delta Q_{m n}+\sum_{m=1}^{M} \int_{\tilde{\Gamma}_{m}}\left(\mathbf{w}_{m} \cdot \delta \mathbf{f}_{m}+\delta \mathbf{w}_{m} \cdot \mathbf{f}_{m}\right) \mathrm{d} \Gamma \\
&+\sum_{m=1}^{M} \sum_{n=1}^{N} \delta \pi_{m n}\left(\oint_{\Gamma_{m n}^{c}} \mathbf{f}_{m} \cdot \mathbf{n} \mathrm{d} \Gamma+Q_{m n}\right) \\
&+\sum_{m=1}^{M} \sum_{n=1}^{N} \pi_{m n}\left(\oint_{\Gamma_{m n}^{c}} \delta \mathbf{f}_{m} \cdot \mathbf{n} \mathrm{d} \Gamma+\delta Q_{m n}\right) .
\end{aligned}
$$

According to Gauss's integral theorem (divergence theorem), 


$$
\iint_{\Omega} \nabla \cdot\left(u_{m} \delta \mathbf{f}_{m}\right) \mathrm{d} \Omega=\int_{\tilde{\Gamma}_{m}} u_{m}\left(\delta \mathbf{f}_{m} \cdot \mathbf{n}\right) \mathrm{d} \Gamma+\sum_{m=1}^{m} \sum_{n=1}^{N} \oint_{\Gamma_{m n}^{c}} u_{m}\left(\delta \mathbf{f}_{m} \cdot \mathbf{n}\right) \mathrm{d} \Gamma,
$$

we can show that

$$
\begin{aligned}
& \delta \Pi=\iint_{\Omega}\left\{\sum_{m=1}^{M}\left(\alpha+\beta\left(\sum_{r=1}^{M}\left|\mathbf{f}_{r}\right|\right)^{\gamma}\right) \frac{1}{\left|\mathbf{f}_{m}\right|} \mathbf{f}_{m}-\nabla u_{m}\right\} \cdot \delta \mathbf{f}_{m} \mathrm{~d} \Omega \\
&+\iint_{\Omega} \sum_{m=1}^{M}\left(u_{m}-D_{m}{ }^{-1}\left(q_{m}\right)\right) \delta q_{m} \mathrm{~d} \Omega \\
&+\iint_{\Omega} \sum_{m=1}^{M}\left(\nabla \cdot \mathbf{f}_{m}+q_{m}\right) \delta u_{m} \mathrm{~d} \Omega \\
&+\sum_{m=1}^{M} \int_{\tilde{\Gamma}_{m}}^{\mathbf{f}_{m}} \cdot \delta \mathbf{w}_{m} \mathrm{~d} \Gamma \\
&+\sum_{m=1}^{M} \sum_{n=1}^{N}\left(\oint_{\Gamma_{m n}^{\mathrm{c}}} \mathbf{f}_{m} \cdot \mathbf{n} \mathrm{d} \Gamma+Q_{m n}\right) \delta \pi_{m n} \\
&+\sum_{m=1}^{M} \sum_{n=1}^{N}\left(U_{m n}+\pi_{m n}\right) \delta Q_{m n} \\
&+\sum_{m=1}^{M} \sum_{n=1}^{N} \oint_{\Gamma_{m n}^{\mathrm{c}}}\left(u_{m}+\pi_{m n}\right) \delta \mathbf{f}_{m} \cdot \mathbf{n} \mathrm{d} \Gamma \\
&+\sum_{m=1}^{M} \int_{\tilde{\Gamma}_{m}}\left(u_{m} \mathbf{n}+\mathbf{w}_{m}\right) \cdot \delta \mathbf{f}_{m} \mathrm{~d} \Gamma
\end{aligned}
$$

As $\delta \mathbf{f}_{m}, \delta q_{m}, \delta u_{m}, \delta \mathbf{w}_{m}, \delta Q_{m n}$, and $\delta \pi_{m n}, n=1,2, \ldots, N_{m}, m=1,2, \ldots, M$, are arbitrary functions in $\Omega$, and $\delta \mathbf{f}_{m}$ vanishes on the boundary $\widetilde{\Gamma}_{m}, m=1,2, \ldots, M$, the stationary point of the Lagrangian requires that

$$
\begin{gathered}
\left(\alpha+\beta\left(\sum_{r=1}^{M}\left|\mathbf{f}_{r}\right|\right)^{\gamma}\right) \frac{\mathbf{f}_{m}}{\left|\mathbf{f}_{m}\right|}-\nabla u_{m}=\mathbf{0}, \quad \forall(x, y) \in \Omega, m=1,2, \ldots, M, \\
u_{m}=D_{m}^{-1}\left(q_{m}\right) \quad \text { or } \quad q_{m}=D\left(u_{m}\right), \quad \forall(x, y) \in \Omega, m=1,2, \ldots, M, \\
\nabla \cdot \mathbf{f}_{m}+q_{m}=0, \quad \forall(x, y) \in \Omega, m=1,2, \ldots, M, \\
\mathbf{f}_{m}=\mathbf{0}, \quad \forall(x, y) \in \tilde{\Gamma}_{m}, m=1,2, \ldots, M, \\
\oint_{\Gamma_{m n}^{c}} \mathbf{f}_{m} \cdot \mathbf{n} \mathrm{d} \Gamma+Q_{m n}=0, \quad n=1,2, \ldots, N_{m}, m=1,2, \ldots, M, \\
U_{m n}+\pi_{m n}=0, \quad n=1,2, \ldots, N_{m}, m=1,2, \ldots, M, \\
u_{m}+\pi_{m n}=0, \quad \forall(x, y) \in \Gamma_{m n}^{\mathrm{c}}, n=1,2, \ldots, N_{m}, m=1,2, \ldots, M .
\end{gathered}
$$

From equations (21) and (22) we obtain $u_{m}=U_{m n}, \forall(x, y) \in \Gamma_{m n}^{\mathrm{c}}, n=1,2, \ldots, N_{m}$, $m=1,2, \ldots, M$. From equations (17) and (18), the demand functions and flow conservation equations are automatically satisfied. Moreover, from equation (16), we can show that the traffic flow vector of commodity $m$ is parallel to the gradient of the Lagrange multiplier $u_{m}$ of that commodity, i.e., $\mathbf{f}_{m} / / \nabla u_{m}, m=1,2, \ldots, M$, and 


$$
c=\alpha+\beta\left(\sum_{r=1}^{M}\left|\mathbf{f}_{r}\right|\right)^{\gamma}=\left|\nabla u_{m}\right|
$$

Therefore, the norm of the gradient of the Lagrange multiplier that is associated with the flow conservation equation is independent of the commodity type, i.e.,

$$
\left|\nabla u_{1}\right|=\left|\nabla u_{2}\right|=\cdots=\left|\nabla u_{M}\right|
$$

For each traveler at location $\mathrm{H}$, the total cost on any used path $p$ from $\mathrm{H}$ to CBD $m$ via $\mathrm{I}_{m n}$ (for the case of $n=1$, which denotes when the traveler is moving directly toward CBD $m$ in the continuum) can be expressed as

$$
\begin{aligned}
C_{m n p}= & U_{m n}+\int_{p} c \mathrm{~d} s=U_{m n}+\int_{p}\left|\nabla u_{m}\right| \mathrm{d} s \\
& =U_{m n}+\int_{p} \nabla u_{m} \cdot \mathbf{d s}=U_{m n}+u_{m}(\mathrm{H})-u_{m}\left(\mathrm{O}_{m}\right)=u_{m}(\mathrm{H})
\end{aligned}
$$

which is independent of the paths used and the interchange chosen. Therefore, the total costs of all used paths are equal. For any unused path $\tilde{p}$ from $\mathrm{H}$ to CBD $m$ via $\mathrm{I}_{m n}$, the total cost is

$$
\begin{aligned}
C_{m n p}= & U_{m n}+\int_{\tilde{p}} c \mathrm{~d} s=U_{m n}+\int_{\tilde{p}}\left|\nabla u_{m}\right| \mathrm{d} s \\
& \geq U_{m n}+\int_{\tilde{p}} \nabla u_{m} \cdot \mathbf{d s}=U_{m n}+u_{m}(\mathrm{H})-u_{m}\left(\mathrm{O}_{m}\right)=u_{m}(\mathrm{H})
\end{aligned}
$$

The inequality in the above derivation occurs because, in some regions along path $\tilde{p}$, the vectors $\nabla u_{m}$ and ds are not parallel, and $\left|\nabla u_{m}\right| \mathrm{d} s>\nabla u_{m}$.ds for some segments $\zeta \in \tilde{p}$. Therefore, for any unused paths, the total cost is greater than or equal to that of the used paths. This satisfies the user equilibrium conditions (Wardrop 1952) when the elements of $\mathbf{U}=\left(U_{m n}, n=1,2, \ldots, N_{m}, m=1,2, \ldots, M\right)$ represent the corresponding equilibrium travel costs in the discrete network. The Lagrange multiplier associated with the flow conservation constraint can be interpreted as the total cost potential (which is the travel cost to an interchange in the continuum plus the equilibrium travel cost from the interchange to the $\mathrm{CBD}$ in the discrete network). The existence and uniqueness of the continuum models were recently studied by Ho et al. (2007).

The optimization program (P1) can be written in the following abstract form:

$$
\mathbf{Q}=\mathbf{F}(\mathbf{U})
$$


For a given set of equilibrium travel costs from the interchanges to all of the CBDs, program $\mathrm{P} 1$ determines the catchment of each interchange and each CBD, which will form the O-D demand in the discrete network.

\subsection{Discrete system}

The user equilibrium problem in the discrete network becomes one of solving the following mathematical program (P2):

$$
\underset{\mathbf{v}}{\operatorname{Minimize}} \bar{Z}(\mathbf{v})=\sum_{a \in A} \int_{0}^{v_{a}} \bar{c}_{a}(\omega) \mathrm{d} \omega=\sum_{a \in A} \bar{\alpha}_{a} v_{a}+\frac{\bar{\beta}_{a}}{\bar{\gamma}+1} v_{a}^{\bar{\gamma}+1}
$$

subject to

$$
\begin{gathered}
Q_{m n}=\sum_{p \in P_{m n}} h_{m n p}, \quad n=1,2, \ldots, N_{m}, m=1,2, \ldots, M, \\
v_{a}=\sum_{m=1}^{M} \sum_{n=2}^{N_{m}} \sum_{p \in P_{m n}} \delta_{m n p a} h_{m n p}, \quad \forall a \in A, \\
h_{m n p} \geq 0, \quad \forall p \in P_{m n}, n=1,2, \ldots, N_{m}, m=1,2, \ldots, M,
\end{gathered}
$$

where $Q_{m n}$ is the O-D demand matrix in the discrete network obtained by evaluating the catchment of the interchanges in equations (7). The satisfaction of the user equilibrium conditions can be found in the work of Sheffi (1985). Denote $\bar{C}_{m n p}$ as the travel cost on path $p$ from interchange location $n$ to $\mathrm{CBD} m$, and $\bar{U}_{m n}$ as the corresponding minimum travel cost, i.e., $\bar{U}_{m n}=\min \left(\bar{C}_{m n p}, \forall p \in P_{m n}\right)$.

The optimization program (P2) can be written in the following abstract form:

$$
\overline{\mathbf{U}}=\mathbf{G}(\mathbf{Q})
$$

For a given O-D matrix, program P2 determines the equilibrium travel costs from the interchanges to the CBDs.

\subsection{Fixed point problem}

From equations (27) and (29) we have

$$
\overline{\mathbf{U}}=\mathbf{G}(\mathbf{F}(\mathbf{U}))
$$


If we can find a mutually consistent $\mathbf{U}^{*}$ such that $\mathbf{U}^{*}=\mathbf{G}\left(\mathbf{F}\left(\mathbf{U}^{*}\right)\right)$, then $\mathbf{U}^{*}$ is said to be a fixed-point solution that satisfies all of the functional relationships and user equilibrium conditions of the combined problem.

\section{SOLUTION ALGORITHM}

\subsection{Continuum system}

The entire city region is first discretized into a set of triangular finite elements. Let $K^{\mathrm{E}}$ and $K^{\mathrm{N}}$ be the numbers of finite elements and finite-element nodes (FENs) in the entire discretized region, and $K_{m}^{\mathrm{O}}$ and $K_{m n}^{\mathrm{I}}$ be the numbers of FENs on $\tilde{\Gamma}_{m}$ and on the boundaries of interchange $n$ heading to $\operatorname{CBD} m, \Gamma_{m n}^{\mathrm{c}}, n=1,2, \ldots, N_{m}, m=1,2, \ldots, M$. Because the cost potential to $\mathrm{CBD} m$ on the boundaries of interchanges $n$ are equal to $U_{m n}, n=1,2, \ldots, N_{m}$, the optimization program P1 can be solved by determining the stationary point of the following modified Lagrangian,

$$
\begin{aligned}
& \bar{\Pi}=-\sum_{m=1}^{M} \sum_{n=1}^{N_{m}} U_{m n} \oint_{\Gamma_{m n}^{c}} \mathbf{f}_{m} \cdot \mathbf{n} \mathrm{d} \Gamma \\
& +\sum_{e=1}^{K^{\mathrm{E}}} \iint_{\Omega_{e}}\left\{\alpha_{e} \sum_{m=1}^{M}\left|\mathbf{f}_{m e}\right|+\frac{\beta_{e}}{\gamma_{e}+1}\left(\sum_{m=1}^{M} \mid \mathbf{f}_{m e}\right)\right)^{\gamma^{+1}} \\
& +\sum_{m=1}^{M}\left(u_{m e}\left(\nabla \cdot \mathbf{f}_{m e}+D\left(u_{m e}\right)\right)\right) \\
& \left.-\sum_{m=1}^{M} \int_{0}^{D\left(u_{m e}\right)} D_{m}{ }^{-1}(\omega) \mathrm{d} \omega \quad\right\} \mathrm{d} \Omega \\
& +\sum_{m=1}^{M} \sum_{k=1}^{K_{m}^{\mathrm{O}}}\left(w_{x m k} \hat{f}_{x m k}+w_{y m k} \hat{f}_{y m k}\right) \\
& +\sum_{m=1}^{M} \sum_{n=1}^{N_{m}} \sum_{k=1}^{K_{m n}^{\mathrm{I}}} \sigma_{m s_{n k}}\left(\hat{u}_{m s_{n k}}-U_{m n}\right)
\end{aligned}
$$

where $\Omega_{e}$ is the subdomain of finite element $e ; \alpha_{e}, \beta_{e}$, and $\gamma_{e}$ are the coefficients of the cost-flow relationship that are assumed to be constant within the finite element; $\mathbf{f}_{m e}=\left(f_{x m e}(x, y), f_{y m e}(x, y)\right)$ and $u_{m e}(x, y)$ are the flow vector and cost potential, respectively, of commodity $m$ in sub-domain $\Omega_{e}, \hat{f}_{x m k}$ and $\hat{f}_{y m k}$ are the corresponding nodal values of the function $\mathbf{f}_{m}(x, y)$ as evaluated at node $k=1,2, \ldots, K_{m}^{\mathrm{O}}, \hat{u}_{m s_{n k}}$ is the nodal value of the function $u_{m}(x, y)$ as evaluated at node $s_{n k}, k=1,2, \ldots, K_{m n}^{\mathrm{I}}, n=1,2, \ldots, N_{m}$, and $\sigma_{m s_{n k}}$ is the corresponding Lagrange multiplier. 
A three-node linear triangle element, the T3 element, was used to approximate the flowvector function, $\mathbf{f}_{m}(x, y)$, and cost potential function, $u_{m}(x, y), m=1,2, \ldots, M$ (Cheung et al., 1996). Within the element, the flow vector and the cost potential can be expressed as

$$
\begin{gathered}
f_{x m}(x, y)=F_{i}(x, y) \hat{f}_{x m i}+F_{j}(x, y) \hat{f}_{x m j}+F_{k}(x, y) \hat{f}_{x m k}, \quad m=1, \ldots, M,(32) \\
f_{y m}(x, y)=F_{i}(x, y) \hat{f}_{y m i}+F_{j}(x, y) \hat{f}_{y m j}+F_{k}(x, y) \hat{f}_{y m k}, \quad m=1, \ldots, M, \text {, (33) } \\
u_{m}(x, y)=F_{i}(x, y) \hat{u}_{m i}+F_{j}(x, y) \hat{u}_{m j}+F_{k}(x, y) \hat{u}_{m k}, \quad m=1, \ldots, M, \quad \text { (34) }
\end{gathered}
$$

where $i, j$, and $k$ are the corner node numbers of the element. $\hat{f}_{x m i}, \hat{f}_{x m j}$, and $\hat{f}_{x m k}\left(\hat{f}_{y m i}\right.$, $\hat{f}_{y m j}$, and $\hat{f}_{y m k}$ ) are the nodal values of the flow intensity in the $x(y)$ direction, and $\hat{u}_{m i}$, $\hat{u}_{m j}$, and $\hat{u}_{m k}$ are the nodal values of the cost potential of commodity $m$. The linear shape functions $F_{i}, F_{j}$, and $F_{k}$ are given by

$$
\begin{aligned}
& F_{i}(x, y)=\frac{1}{2 \Delta}\left(\xi_{i}+\psi_{i} x+\zeta_{i} y\right), \\
& F_{j}(x, y)=\frac{1}{2 \Delta}\left(\xi_{j}+\psi_{j} x+\zeta_{j} y\right), \\
& F_{k}(x, y)=\frac{1}{2 \Delta}\left(\xi_{k}+\psi_{k} x+\zeta_{k} y\right),
\end{aligned}
$$

where

$$
\xi_{i}=x_{j} y_{k}-x_{k} y_{j}, \psi_{i}=y_{j}-y_{k} \zeta_{i}=x_{k}-x_{j},
$$

and $\left(x_{i}, y_{i}\right),\left(x_{j}, y_{j}\right)$, and $\left(x_{k}, y_{k}\right)$ are the coordinates of nodes $i, j$, and $k$, respectively. $\Delta=\left(\psi_{i} \zeta_{j}-\psi_{j} \zeta_{i}\right) / 2$ is the area of the element. From equations (32-38), the spatial derivatives of $f_{x m}(x, y)$ and $f_{y m}(x, y)$ can be obtained by

$$
\begin{array}{ll}
\frac{\partial f_{x m}}{\partial x}=\frac{1}{2 \Delta}\left(\psi_{i} \hat{f}_{x m i}+\psi_{j} \hat{f}_{x m j}+\psi_{k} \hat{f}_{x m k}\right), & m=1, \ldots, M, \\
\frac{\partial f_{y m}}{\partial y}=\frac{1}{2 \Delta}\left(\zeta_{i} \hat{f}_{y m i}+\zeta_{j} \hat{f}_{y m j}+\zeta_{k} \hat{f}_{y m k}\right), & m=1, \ldots, M .
\end{array}
$$

By substituting equations (32-40) into equation (31), the Lagrangian can be expressed as a function of all of the nodal parameters, i.e., $\bar{\Pi}\left(\Psi, \mathbf{U}^{*}\right)$, where

$$
\Psi=\operatorname{Col}(\hat{\mathbf{f}}, \hat{\mathbf{u}}, \hat{\mathbf{w}}, \hat{\sigma}),
$$




$$
\begin{gathered}
\hat{\mathbf{f}}=\operatorname{Col}\left(\hat{f}_{x m k}, \hat{f}_{y m k}, k=1,2, \ldots, K^{\mathrm{N}}, m=1,2, \ldots, M\right), \\
\hat{\mathbf{u}}=\operatorname{Col}\left(\hat{u}_{m k}, k=1,2, \ldots, K^{\mathrm{N}}, m=1,2, \ldots, M\right), \\
\hat{\mathbf{w}}=\operatorname{Col}\left(w_{x m k}, w_{y m k}, k=1,2, \ldots, K_{m}^{\mathrm{O}}, m=1,2, \ldots, M\right), \\
\hat{\boldsymbol{\sigma}}=\operatorname{Col}\left(\sigma_{m s_{n k}}, k=1,2, \ldots, K_{m n}^{\mathrm{I}}, n=1,2, \ldots, N_{m}, m=1,2, \ldots, M\right),
\end{gathered}
$$

are the control variables for a given $\mathbf{U}^{*}$. The stationary point of the Lagrangian, $\Psi^{*}$, can be located by setting $\mathbf{R}\left(\Psi^{*}, \mathbf{U}^{*}\right)=\nabla_{\Psi} \bar{\Pi}\left(\Psi^{*}, \mathbf{U}^{*}\right)=\mathbf{0}$ for a given $\mathbf{U}^{*}$ as follows. Let $\Psi^{0}$ be an approximate solution to the problem. Expanding $\bar{\Pi}\left(\Psi, \mathbf{U}^{*}\right)$ by Taylor's series around point $\Psi^{0}$, we have

$$
\bar{\Pi}\left(\Psi, \mathbf{U}^{*}\right) \cong \bar{\Pi}\left(\Psi^{0}, \mathbf{U}^{*}\right)+\left(\Psi-\Psi^{0}\right)^{\mathrm{T}} \mathbf{R}+\frac{1}{2}\left(\Psi-\Psi^{0}\right)^{\mathrm{T}} \mathbf{H}\left(\Psi-\Psi^{0}\right),
$$

where $\mathbf{R}=\nabla_{\Psi} \bar{\Pi}\left(\Psi^{0}, \mathbf{U}^{*}\right)$ is the residual vector of the first derivatives evaluated at $\Psi^{0}$ and $\mathbf{H}=\nabla_{\Psi, \Psi}^{2} \bar{\Pi}\left(\Psi^{0}, \mathbf{U}^{*}\right)$ is the Hessian matrix evaluated at $\Psi^{0}$. For a stationary point, the derivatives of $\bar{\Pi}\left(\Psi, \mathbf{U}^{*}\right)$ with respect to all variables vanish. Therefore,

$$
\nabla_{\Psi} \bar{\Pi}\left(\Psi, \mathbf{U}^{*}\right)=\mathbf{R}+\mathbf{H}\left(\Psi-\Psi^{0}\right)=\mathbf{0} .
$$

After rearranging, a better solution can be obtained by

$$
\Psi=\Psi^{0}-\mathbf{H}^{-1} \mathbf{R} .
$$

Let the converged solution be $\Psi^{*}$. From the implicit function theorem, the sensitivity of the solution $\Psi^{*}$ with respect to the perturbed parameters $\mathbf{U}$ at $\mathbf{U}^{*}$ can be expressed as (Wong et al., 2006)

$$
\begin{array}{r}
\left.\nabla_{\mathbf{U}} \Psi^{*}\right|_{\mathbf{U}^{*}}=-\left[\nabla_{\Psi} \mathbf{R}\left(\Psi^{*}, \mathbf{U}^{*}\right)\right]^{-1} \nabla_{\mathbf{U}} \mathbf{R}\left(\Psi^{*}, \mathbf{U}^{*}\right) \\
=-\left[\nabla_{\Psi, \Psi}^{2} \bar{\Pi}\left(\Psi^{*}, \mathbf{U}^{*}\right)\right]^{-1} \nabla_{\mathbf{U}, \Psi}^{2} \bar{\Pi}\left(\Psi^{*}, \mathbf{U}^{*}\right),
\end{array}
$$

By substituting the results of equation (49) into equation (27) and using equation (7), we can determine the sensitivity of O-D matrix $\mathbf{Q}$ in the discrete network with respect to the equilibrium travel costs at interchanges $\mathbf{U}$, i.e.,

$$
\nabla_{\mathbf{U}} \mathbf{Q}=\nabla_{\mathbf{U}} \mathbf{F}(\mathbf{U})
$$


Note that the residual vector, $\nabla_{\Psi} \bar{\Pi}(\Psi, \mathbf{U})$, and matrices, $\nabla_{\Psi, \Psi}^{2} \bar{\Pi}(\Psi, \mathbf{U})$ and $\nabla_{\mathbf{U}, \Psi}^{2} \bar{\Pi}(\Psi, \mathbf{U})$, can be obtained explicitly by differentiating equation (31).

\subsection{Discrete system}

Optimization program P2 can be solved by means of the Frank-Wolfe solution algorithm (LeBlanc et al., 1975), and the sensitivity of the O-D travel costs $\overline{\mathbf{U}}$ with respect to the O-D demand $\mathbf{Q}$ can then be obtained by the method of Tobin and Friesz (1988), i.e.,

$$
\nabla_{\mathbf{Q}} \overline{\mathbf{U}}=\nabla_{\mathbf{Q}} \mathbf{G}(\mathbf{Q}) \text {. }
$$

\subsection{Newtonian algorithm}

The fixed-point problem in equation (30) can now be solved by a Newtonian algorithm as follows. The problem is to find a $\mathbf{U}$ such that

$$
\mathbf{E}(\mathbf{U})=\mathbf{U}-\mathbf{G}(\mathbf{F}(\mathbf{U}))=\mathbf{0},
$$

where $\mathbf{E}(\mathbf{U})$ is a column vector that measures the discrepancies in the O-D travel costs at the interchanges from the two optimization subprograms for the continuum and discrete systems. Let $\mathbf{U}^{*}$ be an approximate solution to the problem. Expanding $\mathbf{E}(\mathbf{U})$ by Taylor's series around point $\mathbf{U}^{*}$, we have

$$
\mathbf{E}(\mathbf{U}) \cong \mathbf{E}\left(\mathbf{U}^{*}\right)+\left\lfloor\nabla_{\mathbf{U}} \mathbf{E}\left(\mathbf{U}^{*}\right)\right\rfloor\left(\mathbf{U}-\mathbf{U}^{*}\right),
$$

where $\nabla_{\mathbf{U}} \mathbf{E}\left(\mathbf{U}^{*}\right)$ is the Jacobian matrix evaluated at $\mathbf{U}^{*}$. For a stationary point, the derivatives of $\mathbf{E}(\mathbf{U})$ with respect to all variables vanish. Therefore, a better solution can be obtained by

$$
\mathbf{U}=\mathbf{U}^{*}-\left[\nabla_{\mathbf{U}} \mathbf{E}\left(\mathbf{U}^{*}\right)\right]^{-1} \mathbf{E}\left(\mathbf{U}^{*}\right)
$$

where $\mathbf{E}\left(\mathbf{U}^{*}\right)$ is evaluated by equation (52), which requires the solutions of the two optimization subproblems for the continuum and discrete systems, and the Jacobian matrix is obtained from the sensitivity analyses of the two subproblems,

$$
\nabla_{\mathbf{U}} \mathbf{E}\left(\mathbf{U}^{*}\right)=\mathbf{I}-\left[\nabla_{\mathbf{Q}} \mathbf{G}\left(\mathbf{Q}^{*}\right) \mid \nabla_{\mathbf{U}} \mathbf{F}\left(\mathbf{U}^{*}\right)\right] \text {. }
$$


where $\mathbf{Q}^{*}=\mathbf{F}\left(\mathbf{U}^{*}\right)$, and $\nabla_{\mathbf{U}} \mathbf{F}\left(\mathbf{U}^{*}\right)$ and $\nabla_{\mathbf{Q}} \mathbf{G}\left(\mathbf{Q}^{*}\right)$ are obtained by equations (50) and (51), respectively.

\subsection{Solution procedure}

The solution procedure is summarized as follows.

Step 1. Set $k=0$. Set the travel costs on all links as free-flowing costs $\left(\bar{\alpha}_{a}, \forall a \in A\right)$, and find the shortest paths from all interchanges to each CBD. Set the minimum path costs as $\mathbf{U}^{(k)}$. Find an initial solution $\Psi^{*}$.

Step 2. Solve optimization subproblem P1 for the continuum system.

Step 2.1. Set $l=0$. Set $\Psi^{(l)}=\Psi^{*}$.

Step 2.2. Evaluate $\mathbf{R}\left(\Psi^{(l)}\right)$ and $\mathbf{H}\left(\Psi^{(l)}\right)$.

Step 2.3. If $\left|\mathbf{R}\left(\Psi^{(l)}\right)\right|<\varepsilon_{1}$, an acceptable error, then set $\Psi^{*}=\Psi^{(l)}$ and go to Step 2.6 .

Step 2.4. Otherwise, compute $\mathbf{Y}^{(l)}$ by solving the set of simultaneous equations $\mathbf{H}\left(\Psi^{(l)}\right) \mathbf{Y}^{(l)}=\mathbf{R}\left(\Psi^{(l)}\right)$ and evaluate $\Psi^{(l+1)}=\Psi^{(l)}-\mathbf{Y}^{(l)}$.

Step 2.5. Set $l=l+1$ and go to Step 2.2.

Step 2.6. Determine the O-D demand matrix $\mathbf{Q}^{(k)}$ by substituting the solution $\Psi^{*}$ in equation (7).

Step 2.7. Conduct sensitivity analysis to determine $\nabla_{\mathbf{U}} \mathbf{F}\left(\mathbf{U}^{(k)}\right)$.

Step 3. Solve optimization sub-problem P2 for the discrete system to obtain the minimum equilibrium path costs $\overline{\mathbf{U}}^{(k)}=\mathbf{G}\left(\mathbf{Q}^{(k)}\right)$. Conduct sensitivity analysis to determine $\nabla_{\mathbf{Q}} \mathbf{G}\left(\mathbf{Q}^{(k)}\right)$.

Step 4. Evaluate $\mathbf{E}^{(k)}=\mathbf{U}^{(k)}-\overline{\mathbf{U}}^{(k)}$ and $\nabla_{\mathbf{U}} \mathbf{E}\left(\mathbf{U}^{(k)}\right)=\mathbf{I}-\left[\nabla_{\mathbf{Q}} \mathbf{G}\left(\mathbf{Q}^{(k)}\right)\left[\nabla_{\mathbf{U}} \mathbf{F}\left(\mathbf{U}^{(k)}\right)\right]\right.$.

Step 5. If $\left|\mathbf{E}^{(k)}\right|<\varepsilon_{2}$, an acceptable error, then stop and $\mathbf{U}^{*}=\mathbf{U}^{(k)}, \mathbf{Q}^{*}=\mathbf{Q}^{(k)}$, and $\Psi^{*}$ are the solution.

Step 6. Otherwise, compute $\mathbf{T}^{(k)}$ by solving the set of simultaneous equations $\left[\nabla_{\mathbf{U}} \mathbf{E}\left(\mathbf{U}^{(k)}\right)\right] \mathbf{T}^{(k)}=\mathbf{E}^{(k)}$ and evaluate $\mathbf{U}^{(k+1)}=\mathbf{U}^{(k)}-\mathbf{T}^{(k)}$. 
Step 7. Set $k=k+1$ and go to Step 2 .

\section{EXAMPLE}

We consider an example city with two CBDs, seven interchanges, and 30 freeway links, as shown in Figure 2. The CBDs are located at $\mathrm{O}_{1}$ and $\mathrm{O}_{2}$, and the interchanges are at $\mathrm{I}_{1}, \mathrm{I}_{2}$, $\ldots, \mathrm{I}_{7}$. The travel demand functions are

$$
\begin{aligned}
& q_{1}=40 \exp \left(-0.5 u_{1}\right) \\
& q_{2}=60 \exp \left(-0.5 u_{2}\right)
\end{aligned}
$$

everywhere in the city, where $q_{1}$ and $q_{2}$ are measured in veh/h/km², and $u_{1}$ and $u_{2}$ are measured in hours. The cost-flow relationship in the continuum system is specified as

$$
c=0.01+0.1 \times 10^{-3}\left(\left|\mathbf{f}_{1}\right|+\left|\mathbf{f}_{2}\right|\right)^{1.2}
$$

throughout the city, where $c$ is measured in $\mathrm{h} / \mathrm{km}$, and $\left|\mathbf{f}_{1}\right|$ and $\left|\mathbf{f}_{2}\right|$ are measured in veh/h/km. The cost-flow relationship for the links in the discrete system is specified as

$$
\bar{c}_{a}=\bar{c}_{a 0}\left(1.0+2.62\left(\frac{v_{a}}{C_{a}}\right)^{5}\right)
$$

where $\bar{c}_{a}$ and $v_{a}$ are the travel cost in hours and the traffic flow in veh/h on link $a$, respectively, and $\bar{c}_{a 0}$ and $C_{a}$ are the free-flowing travel cost in hours and the practical capacity in veh/h on link $a$, respectively. The values adopted in the example calculation are listed in Table I. The finite-element discretization of the continuum system is shown in Figure 3.

The maximum acceptable errors $\varepsilon_{1}$ and $\varepsilon_{2}$ for the solution algorithm are set at $0.1 \%$. The convergence characteristics of the solution algorithm are shown in Figure 4. The solution converges very rapidly in the first few iterations and satisfies the convergence criteria after 39 iterations. The results of the sensitivity analyses provide a good indication of the search direction leading to the fixed-point solution. The flow vectors to CBD 1 are plotted in Figure 5 , from which the path from a home location to the CBD or interchanges can be traced by locating the streamline in the diagram. Similar results can also be plotted for the traffic flows to CBD 2. The catchment areas of the CBDs or interchanges can also be observed from the directions in which the travelers are moving. The traffic flows in the city's continuum and 
discrete networks are shown in Figure 6. The traffic flows in the discrete network are also tabulated in the last two columns of Table I. Many links in the discrete network are not used, and heavy traffic flows are present on the direct links between the two CBDs. Traffic congestion is observed at the CBDs and at all of the interchanges.

[Figure 2 to be inserted here]

[Figure 3 to be inserted here]

[Figure 4 to be inserted here]

[Figure 5 to be inserted here]

[Figure 6 to be inserted here]

[Table I to be inserted here]

Figures 7 and 8 show the contour plots of the total travel cost, and Figures 9 and 10 show the travel demands from the home locations to the CBDs. The travel demand generally decreases along with the distance from the CBDs. However, it is interesting to note that the travel demand increases locally when approaching any of the interchanges to take advantage of the high-speed movement on the discrete network. This also reflects the realistic situation that high development potentials are usually concentrated at major freeway interchanges. For this example, the total demand generated is 40,499 veh/h, with 16,420 for CBD 1 and 24,079 for CBD 2. The catchments of traffic to the CBDs and interchanges are shown in Table II. Approximately $30 \%$ of the traffic travels directly to the CBDs within the continuum, whereas the rest uses the freeway system via the interchanges. The cost potentials at the CBDs and at the interchanges for different commodities are also listed in the table.

[Figure 7 to be inserted here]

[Figure 8 to be inserted here]

[Figure 9 to be inserted here] 
[Figure 10 to be inserted here]

[Table II to be inserted here]

\section{CONCLUSIONS}

We developed a multi-commodity traffic equilibrium model for the discrete/continuum transportation system in which we consider a city with several highly compact CBDs. In this city, the commuters' origins are continuously dispersed and the travel demand depends on the total travel cost to the CBD. The transportation system is divided into two layers: major freeways and the dense network of surface streets. Whereas the major freeway network is modeled with the conventional discrete-network approach, the dense network of surface streets is approximated as a continuum. The travelers to each CBD can either move within the continuum (surface streets) and then transfer to the discrete network (freeways) at an interchange (ramp) before moving to the CBD on the discrete network, or they can travel directly to the CBD within the continuum. Specific travel cost-flow relationships for the two layers of transportation facilities have been considered. In the model, the commuters follow a user equilibrium route choice pattern in which, for each O-D pair, no traveler can reduce his or her individual travel cost to the destination (CBD) by unilaterally changing routes.

The problem has been formulated as a simultaneous optimization program with two subproblems. One sub-problem is a conventional traffic assignment problem from the interchanges to the CBDs in the discrete network, and the other is a traffic assignment problem with multiple commodities in the continuum system. This simultaneous optimization program belongs to the class of fixed-point problems. Based on the sensitivity analyses of the two sub-problems, a Newtonian algorithm is proposed to solve the resultant simultaneous optimization program. The advantages of the formulation and solution algorithm are that we can make use of state-of-the-art developments in continuum and discrete network modeling techniques and recent advances in sensitivity analyses in transportation modeling. A numerical example has been given to demonstrate the effectiveness of the proposed method. This multi-commodity traffic equilibrium model provides a solid theoretical foundation for future applications of the discrete/continuum transportation system, for example, facility competition problems, such as airport competition and freeway network planning. The use of 
a discrete/continuum approach can also help to intuitively identify the sensitivity of the travel cost of the catchment of each interchange or CBD. This will provide insightful information that can be used to inform the future formulation of a detailed cordon scheme within an urban area with an elevated expressway network.

\section{ACKNOWLEDGEMENTS}

The work described in this paper was supported by grants from the Research Grants Council of the Hong Kong Special Administrative Region, China (Project No.: 701500 and 17208614), and the National Natural Science Foundation of China (No. 71371143).

\section{REFERENCES}

Beckmann, M., 1952. A continuous model of transportation. Econometrica, 20, 643-660.

Boyce, D.E., 1984. Urban transportation network-equilibrium and design models: recent achievements and future prospects. Environment and Planning A, 16, 1445-1474.

Boyce, D.E. and Daskin, M.S., 1997. Urban transportation. In C. Revelle and A. McGarity (eds), Design and Operation of Civil and Environmental Engineering Systems. New York: Wiley, pp. 277-341.

Buckley, D.J., 1979. Traffic assignment in a two-dimensional continuous representation of a traffic network with flow-dependent speeds. Transportation Research Part B, 13, 167-179.

Cheung, Y.K., Lo, S.H., and Leung, A.Y.T., 1996. Finite Element Implementation. Blackwell Science, Oxford, England.

Dafermos, S.C., 1980. Continuum modeling of transportation networks. Transportation Research Part B, 14, 295-301.

D’Este, G., 1987. Trip assignment to radial major roads. Transportation Research Part B, 21, 433-442.

Du J., Wong S.C., Shu C.W., Xiong T., Zhang M., and Choi K., 2013. Revisiting Jiang's dynamic continuum model for urban cities. Transportation Research Part B, 56, 96-119.

Fernandez, L.J.E. and Freisz, T.L., 1983. Equilibrium predictions in transportation markets: the state of the art. Transportation Research Part B, 17, 155-172.

Ho, H.W. and Wong, S.C., 2005. A combined model of housing location and traffic equilibrium problems in a continuous transportation system. In Hani S. Mahmassani (ed.) 
Transportation and Traffic Theory: Flow, Dynamics and Human Interaction. Elsevier Science Ltd., pp. 761-780, USA.

Ho, H.W. and Wong, S.C., 2007. Housing allocation problem in a continuum transportation system. Transportmetrica, 3(1), 21-39.

Ho, H.W., Wong, S.C., and Loo, B.P.Y., 2003. A continuous traffic equilibrium model with multiple user classes. Journal of the Eastern Asia Society for Transportation Studies, 5, 2831-2845.

Ho, H.W., Wong, S.C., and Loo, B.P.Y., 2004. Sequential optimization approach for the multi-class user equilibrium problem in a continuous transportation system. Journal of Advanced Transportation, 38, 323-345.

Ho, H.W., Wong, S.C., Yang, H., and Loo B.P.Y., 2005. Cordon-based congestion pricing in a continuum traffic equilibrium system. Transportation Research Part A, 39, 813-834.

Ho, H.W., Wong, S.C., and Loo, B.P.Y., 2006. Combined distribution and assignment model for a continuum traffic equilibrium problem with multiple user classes. Transportation Research Part B, 40, 633-650.

Ho, H.W., Wong, S.C., and Hau, T.D., 2007. Existence and uniqueness of a solution for the multi-class user equilibrium problem in a continuum transportation system. Transportmetrica, 3, 107-117.

Ho, H.W., Wong, S.C., and Sumalee, A., 2013. A congestion-pricing problem with a polycentric region and multi-class users: a continuum modelling approach. Transportmetrica A: Transport Science, 9(6), 514-545.

Jiang Y.Q., Wong S.C., Ho H.W., Zhang P., Liu R.X., and Sumalee A., 2011. A dynamic traffic assignment model for a continuum transportation system. Transportation Research Part B, 45, 343-363.

Lam, T.N. and Newell, G.F., 1967. Flow dependent traffic assignment on a circular city. Transportation Science, 1, 318-361.

LeBlanc, L.J., Morlok, E.K., and Pierskalla, W., 1975. An efficient approach to solving the road network equilibrium traffic assignment problem. Transportation Research, 9, 309318.

Loo, B.P.Y., Ho, H.W., and Wong, S.C., 2005. An application of the continuous equilibrium modelling approach in understanding the geography of air passenger flows in a multiairport region. Applied Geography, 25, 169-199.

Puu, T., 1977. A proposed definition of traffic flow in continuous transportation models. Environment and Planning A, 9, 559-567. 
Sasaki, T., Iida, Y., and Yang, H., 1990. User equilibrium traffic assignment by continuum approximation of network flow. Proceedings of the 11th International Symposium on Transportation and Traffic Theory, Japan, Yokohama, July, pp. 233-252.

Sheffi, Y., 1985. Urban Transportation Networks. Englewood Cliffs, N.J.: Prentice-Hall.

Taguchi, A. and Iri, M., 1982. Continuum approximation to dense networks and its application to the analysis of urban road networks. Mathematical Programming Study, 20, 178-217.

Tao Y.Z., Jiang Y.Q., Du J., Wong S.C., Zhang P., Xia Y.H., and Choi K., 2014. Dynamic system-optimal traffic assignment for a city using the continuum modeling approach. Journal of Advanced Transportation, 48, 782-797.

Tobin, R.L. and Friesz, T.L., 1988. Sensitivity analysis for equilibrium network flows. Transportation Science, 22, 242-250.

Vaughan, R.J., 1987. Urban Spatial Traffic Patterns. London, Pion.

Wardrop, J.G., 1952. Some theoretical aspects of road traffic research. Proceedings of the Institution of Civil Engineers Part II, 1, 325-378.

Wardrop, J.G., 1971. Minimum cost paths when the cost per unit length depends on location and direction. Fifth Symposium on Transportation and Traffic Flow. Berkeley, Calif., pp. 429-437.

Williams, H.C.W.L. and Ortuzar, J.D., 1976. Some generalizations and applications of the velocity field concept: trip patterns in idealized cities. Transportation Research, 10, 6573.

Wong, S.C., 1994. An alternative formulation of D’Este's trip assignment model. Transportation Research Part B, 28, 187-196.

Wong, S.C., 1998. Multi-commodity traffic assignment by continuum approximation of network flow with variable demand. Transportation Research Part B, 32, 567-581.

Wong, S.C. and Sun S.H., 2001. A combined distribution and assignment model for continuous facility location problem. The Annals of Regional Science, 35, 267-281.

Wong, S.C. and Yang, H., 1999. Determining market areas captured by competitive facilities: a continuous equilibrium modeling approach. Journal of Regional Science, 39, 51-72.

Wong, S.C., Lee, C.K., and Tong, C.O., 1998. Finite element solution for the continuum traffic equilibrium problems. International Journal for Numerical Methods in Engineering, 43, 1253-1273. 
Wong, S.C., Du, Y.C., Ho, H.W., and Sun, L.J., 2003. A simultaneous optimization formulation of the discrete/continuous transportation system. Transportation Research Record, 1857, 11-20.

Wong, S.C., Zhou, C.W., Lo, H.K., and Yang, H., 2004. An improved solution algorithm for the multi-commodity continuous distribution and assignment model. Journal of Urban Planning and Development-ASCE, 130, 14-23.

Wong, S.C., Du, Y.C., Sun, L.J., and Loo, B.P.Y., 2006. Sensitivity analysis for a continuum traffic equilibrium problem. The Annals of Regional Science, 40, 493-514.

Yang, H. and Wong, S.C., 2000. A continuous equilibrium model for estimating market areas by competitive facilities with elastic demand and market externality. Transportation Science, 34, 216-227.

Yang, H., Yagar, S., and Iida, Y., 1994. Traffic assignment in a congested discrete/continuous transportation system. Transportation Research Part B, 28, 161-174.

Yin J., Wong S.C., and Sze N.N., 2012. Optimization of housing allocation and transport emissions using continuum modeling approach. Asian Transport Studies, 2, 93-108.

Yin J., Wong S.C., Sze N.N., and Ho H.W., 2013. A continuum model for housing allocation and transportation emission problems in a polycentric city. International Journal of Sustainable Transportation, 7, 275-298.

Zienkiewicz, O.C. and Taylor, R.L., 1989. The Finite Element Method. McGraw-Hill International Editions.

Zitron, N.R., 1974. A continuous model of optimal-cost routes in a circular city. Journal of Optimisation Theory and Applications, 14, 291-303. 


\section{TABLES AND FIGURES}

Table I. The input and output data of the discrete network.

Table II. The catchment of CBDs and interchanges.

Figure $1 . \quad$ The discrete/continuum transportation system.

Figure 2. The example city.

Figure 3. The finite-element discretization of the continuum of the city.

Figure 4. The convergence characteristics of the solution algorithm.

Figure 5. The vector plot of traffic movements to CBD 1 within the continuum of the city.

Figure 6. The traffic flows in the continuum and the freeway network.

Figure 7. The contour plot of the total travel cost to CBD 1 in the city.

Figure 8. The contour plot of the total travel cost to CBD 2 in the city.

Figure 9. The travel demand to CBD 1 in the city.

Figure 10. The travel demand to CBD 2 in the city. 
TABLE I.

THE INPUT AND OUTPUT DATA OF THE DISCRETE NETWORK.

\begin{tabular}{|c|c|c|c|c|c|r|c||}
\hline \multicolumn{3}{|c|}{ Link } & \multicolumn{2}{c|}{ Input } & \multicolumn{2}{c|}{ Output } \\
\hline No. & Start & End & $\begin{array}{c}\text { Length } \\
(\mathrm{km})\end{array}$ & $\begin{array}{c}\mathrm{C}_{a} \\
(\mathrm{veh} / \mathrm{h})\end{array}$ & $\begin{array}{c}\bar{c}_{a 0} \\
(\mathrm{~min})\end{array}$ & $\begin{array}{c}v_{a} \\
\text { (veh/h) }\end{array}$ & $\begin{array}{c}\bar{c}_{a} \\
(\mathrm{~min})\end{array}$ \\
\hline 1 & $\mathrm{O}_{1}$ & $\mathrm{O}_{2}$ & 10.3 & 8,000 & 6.2 & 5,939 & 9.86 \\
\hline 2 & $\mathrm{O}_{2}$ & $\mathrm{O}_{1}$ & 10.3 & 8,000 & 6.2 & 3,429 & 6.43 \\
\hline 3 & $\mathrm{O}_{1}$ & $\mathrm{I}_{1}$ & 8.5 & 6,000 & 6.4 & 0 & 6.40 \\
\hline 4 & $\mathrm{I}_{1}$ & $\mathrm{O}_{1}$ & 8.5 & 6,000 & 6.4 & 2,624 & 6.67 \\
\hline 5 & $\mathrm{O}_{1}$ & $\mathrm{I}_{2}$ & 5.0 & 6,000 & 5.0 & 0 & 5.00 \\
\hline 6 & $\mathrm{I}_{2}$ & $\mathrm{O}_{1}$ & 5.0 & 6,000 & 5.0 & 3,245 & 5.61 \\
\hline 7 & $\mathrm{O}_{1}$ & $\mathrm{I}_{5}$ & 8.0 & 6,000 & 6.0 & 0 & 6.00 \\
\hline 8 & $\mathrm{I}_{5}$ & $\mathrm{O}_{1}$ & 8.0 & 6,000 & 6.0 & 1,776 & 6.04 \\
\hline 9 & $\mathrm{O}_{1}$ & $\mathrm{I}_{6}$ & 6.3 & 6,000 & 4.7 & 0 & 4.70 \\
\hline 10 & $\mathrm{I}_{6}$ & $\mathrm{O}_{1}$ & 6.3 & 6,000 & 4.7 & 2,411 & 4.83 \\
\hline 11 & $\mathrm{O}_{1}$ & $\mathrm{I}_{7}$ & 9.5 & 6,000 & 7.1 & 0 & 7.10 \\
\hline 12 & $\mathrm{I}_{7}$ & $\mathrm{O}_{1}$ & 9.5 & 6,000 & 7.1 & 3,599 & 8.54 \\
\hline 13 & $\mathrm{O}_{2}$ & $\mathrm{I}_{2}$ & 8.0 & 6,000 & 6.0 & 0 & 6.00 \\
\hline 14 & $\mathrm{I}_{2}$ & $\mathrm{O}_{2}$ & 8.0 & 6,000 & 6.0 & 3,914 & 7.86 \\
\hline 15 & $\mathrm{O}_{2}$ & $\mathrm{I}_{3}$ & 8.3 & 6,000 & 6.2 & 0 & 6.20 \\
\hline 16 & $\mathrm{I}_{3}$ & $\mathrm{O}_{2}$ & 8.3 & 6,000 & 6.2 & 2,940 & 6.66 \\
\hline 17 & $\mathrm{O}_{2}$ & $\mathrm{I}_{4}$ & 7.1 & 6,000 & 5.3 & 0 & 5.30 \\
\hline 18 & $\mathrm{I}_{4}$ & $\mathrm{O}_{2}$ & 7.1 & 6,000 & 5.3 & 4,535 & 8.73 \\
\hline 19 & $\mathrm{O}_{2}$ & $\mathrm{I}_{5}$ & 6.7 & 6,000 & 5.0 & 0 & 5.00 \\
\hline 20 & $\mathrm{I}_{5}$ & $\mathrm{O}_{2}$ & 6.7 & 6,000 & 5.0 & 4,341 & 7.60 \\
\hline 21 & $\mathrm{I}_{1}$ & $\mathrm{I}_{2}$ & 11.5 & 4,000 & 8.6 & 1,266 & 8.67 \\
\hline 22 & $\mathrm{I}_{2}$ & $\mathrm{I}_{1}$ & 11.5 & 4,000 & 8.6 & 0 & 8.60 \\
\hline 23 & $\mathrm{I}_{2}$ & $\mathrm{I}_{3}$ & 9.9 & 4,000 & 7.4 & 0 & 7.40 \\
\hline 24 & $\mathrm{I}_{3}$ & $\mathrm{I}_{2}$ & 9.9 & 4,000 & 7.4 & 1,352 & 7.49 \\
\hline 25 & $\mathrm{I}_{3}$ & $\mathrm{I}_{4}$ & 8.7 & 4,000 & 6.5 & 0 & 6.50 \\
\hline 26 & $\mathrm{I}_{4}$ & $\mathrm{I}_{3}$ & 8.7 & 4,000 & 6.5 & 0 & 6.50 \\
\hline 27 & $\mathrm{I}_{4}$ & $\mathrm{I}_{5}$ & 12.3 & 4,000 & 9.2 & 0 & 9.20 \\
\hline 28 & $\mathrm{I}_{5}$ & $\mathrm{I}_{4}$ & 12.3 & 4,000 & 9.2 & 0 & 9.20 \\
\hline 29 & $\mathrm{I}_{5}$ & $\mathrm{I}_{6}$ & 9.2 & 4,000 & 6.9 & 0 & 6.90 \\
\hline 30 & $\mathrm{I}_{6}$ & $\mathrm{I}_{5}$ & 9.2 & 4,000 & 6.9 & 1,614 & 7.09 \\
\hline & & & & & & & \\
\hline \hline
\end{tabular}


TABLE II.

THE CATCHMENT OF CBDS AND INTERCHANGES.

\begin{tabular}{|c|c|c|c|c|c||}
\hline \multirow{2}{*}{$\begin{array}{c}\text { CBD / } \\
\text { Interchange }\end{array}$} & \multicolumn{2}{|c|}{ Traffic to CBD 1 } & \multicolumn{2}{c|}{ Traffic to CBD 2 } & \multirow{2}{*}{$\begin{array}{c}\text { Total flow } \\
\text { (veh/h) }\end{array}$} \\
\cline { 2 - 5 } & Flow (veh/h) & Cost (min) & Flow (veh/h) & Cost (min) & 5,275 \\
\hline $\mathrm{O}_{1}$ & 3,011 & 0.00 & 2,264 & 9.86 & 5,839 \\
\hline $\mathrm{O}_{2}$ & 1,513 & 6.43 & 4,326 & 0.00 & 3,890 \\
\hline $\mathrm{I}_{1}$ & 1,615 & 6.67 & 2,275 & 16.53 & 4,541 \\
\hline $\mathrm{I}_{2}$ & 1,893 & 5.61 & 2,648 & 7.86 & 4,292 \\
\hline $\mathrm{I}_{3}$ & 1,503 & 13.09 & 2,789 & 6.66 & 4,536 \\
\hline $\mathrm{I}_{4}$ & 1,766 & 15.16 & 2,770 & 8.73 & 4,502 \\
\hline $\mathrm{I}_{5}$ & 1,776 & 6.04 & 2,726 & 7.60 & 4,025 \\
\hline $\mathrm{I}_{6}$ & 1,823 & 4.83 & 2,202 & 14.69 & 3,599 \\
\hline $\mathrm{I}_{7}$ & 1,520 & 8.54 & 2,079 & 18.40 & 40,499 \\
\hline Total & 16,420 & - & 24,079 & - & \\
\hline \hline
\end{tabular}


(a) Combined

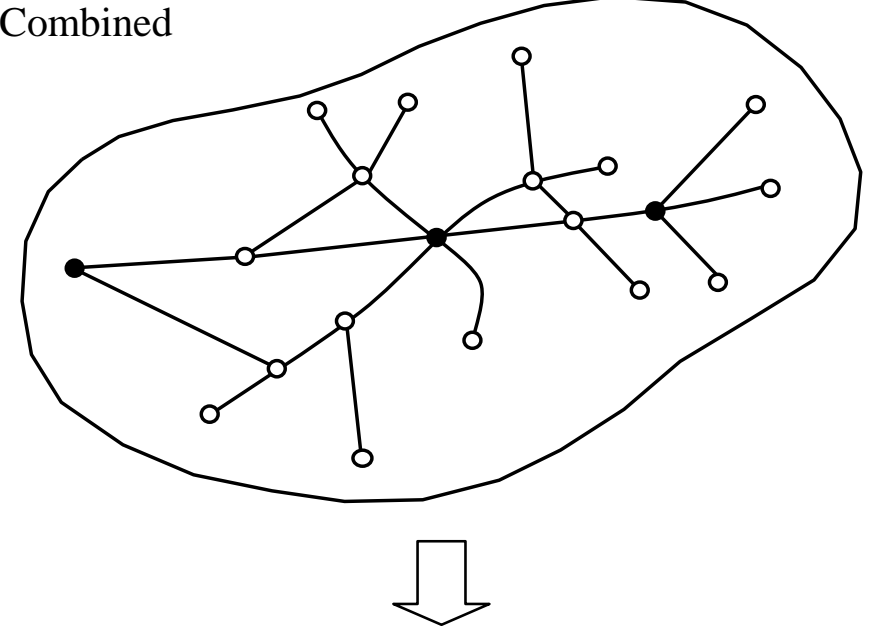

(b) Discrete

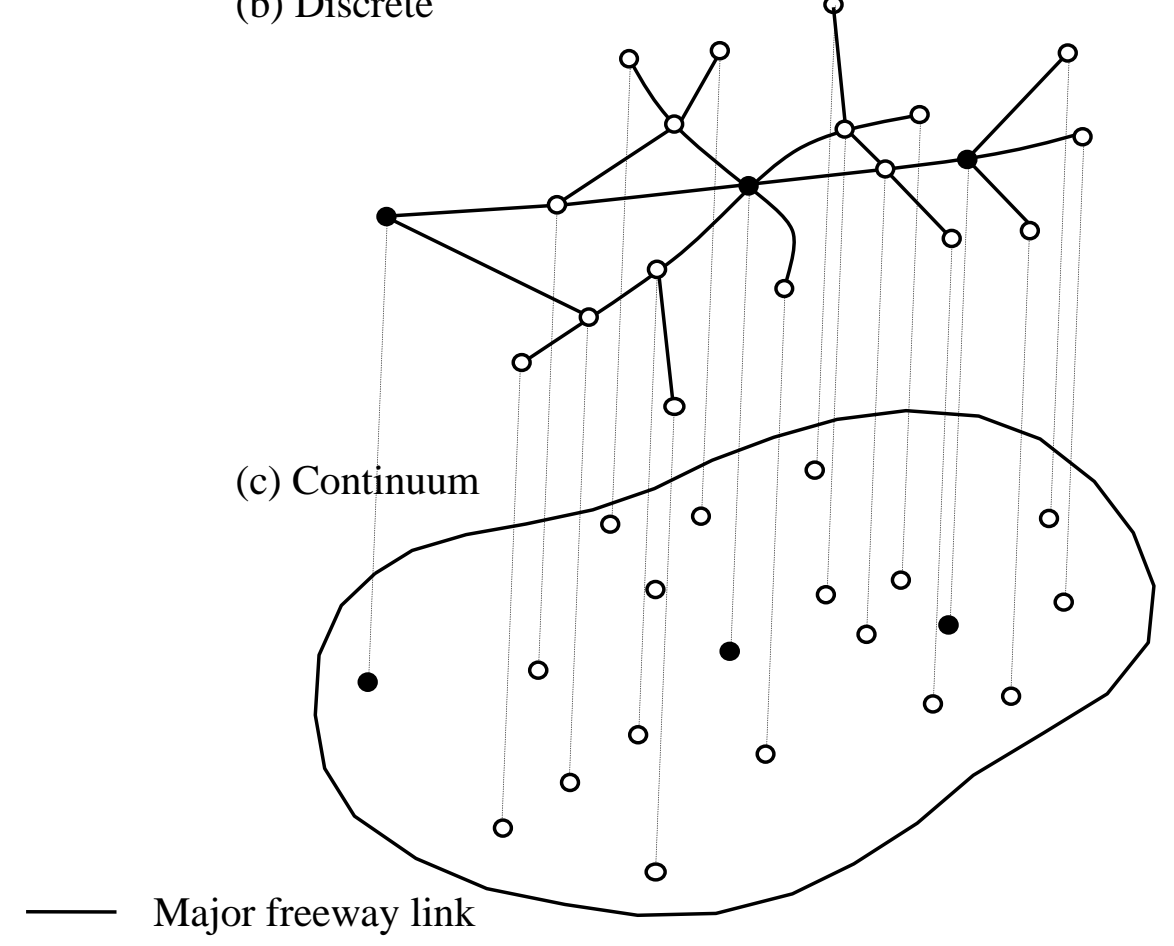

- Central business district

○ Major interchange

Figure 1. The discrete/continuum transportation system. 


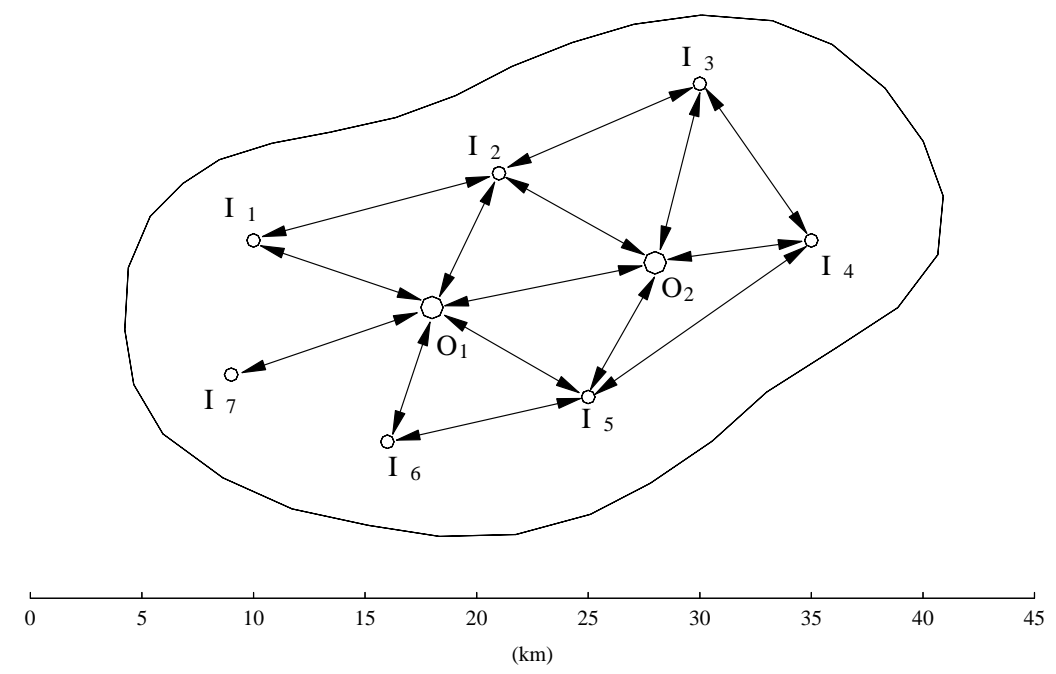

Figure 2. The example city. 


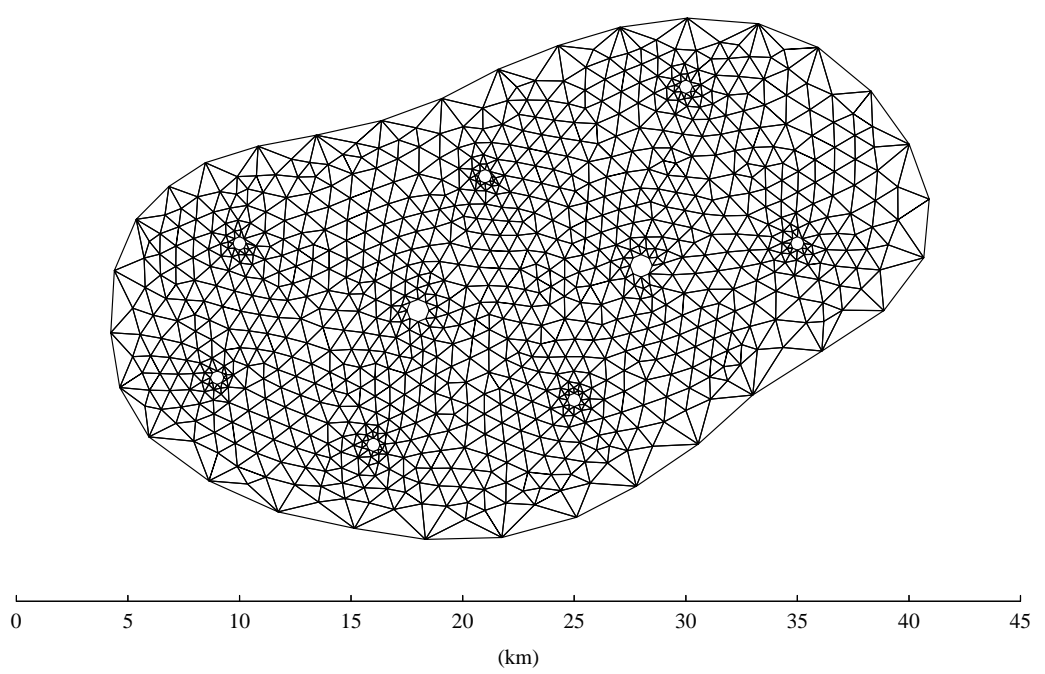

Figure 3. The finite-element discretization of the continuum of the city. 


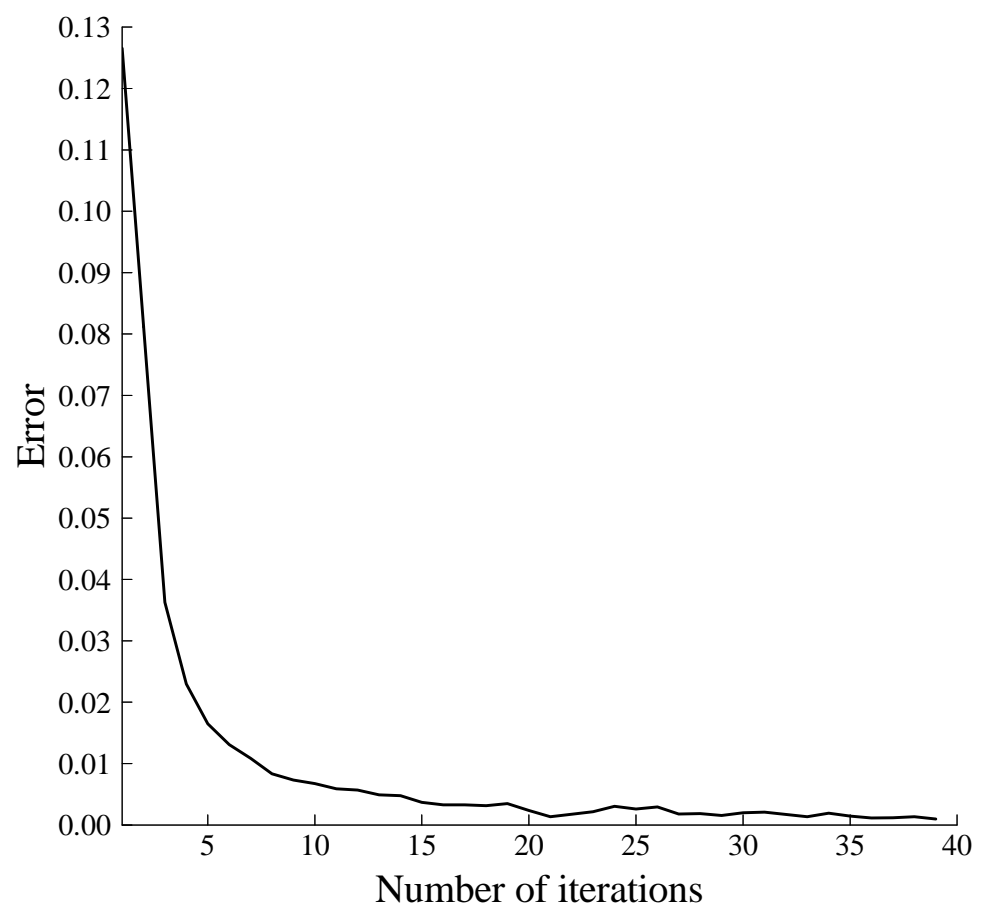

Figure 4. The convergence characteristics of the solution algorithm. 


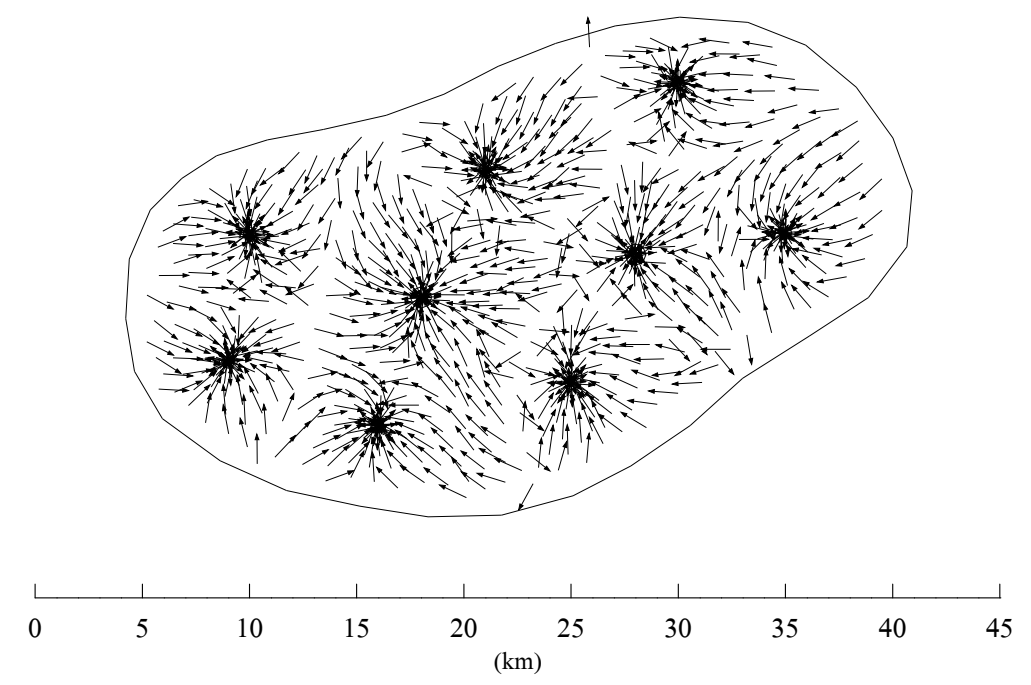

Figure 5. The vector plot of traffic movements to CBD 1 within the continuum of the city. 


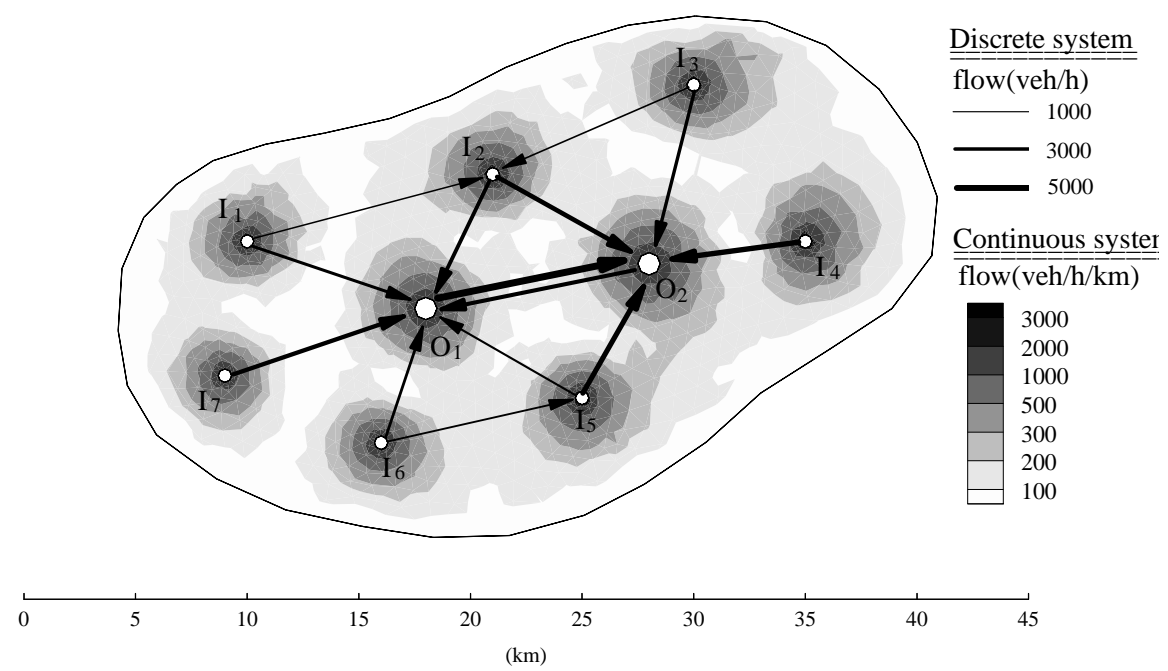

Figure 6. The traffic flows in the continuum and the freeway network. 


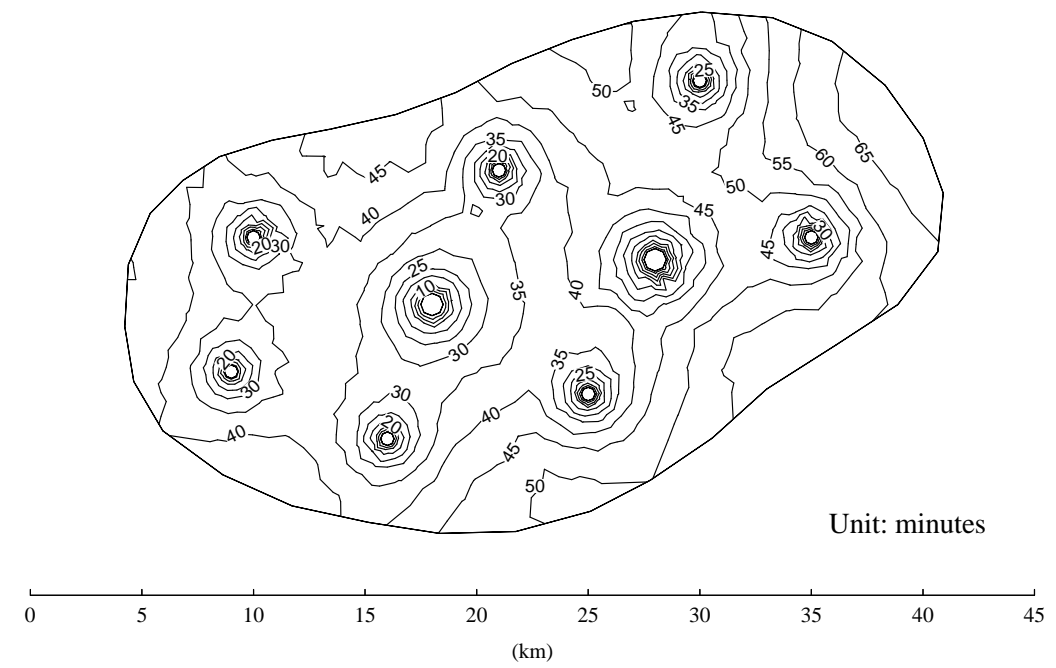

Figure 7. The contour plot of the total travel cost to CBD 1 in the city. 


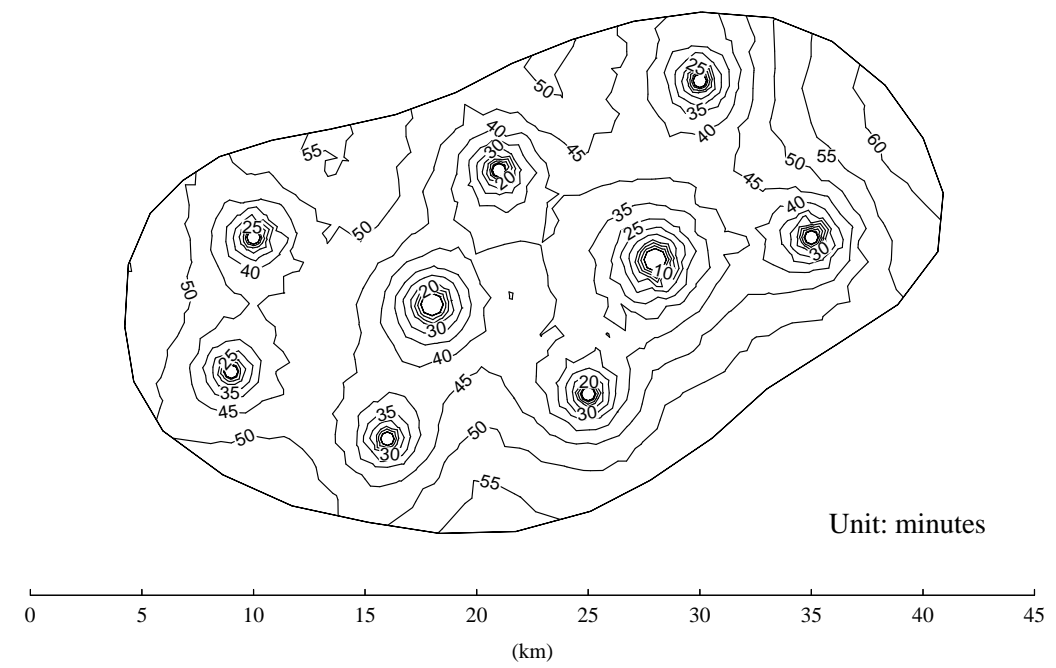

Figure 8 . The contour plot of the total travel cost to CBD 2 in the city 


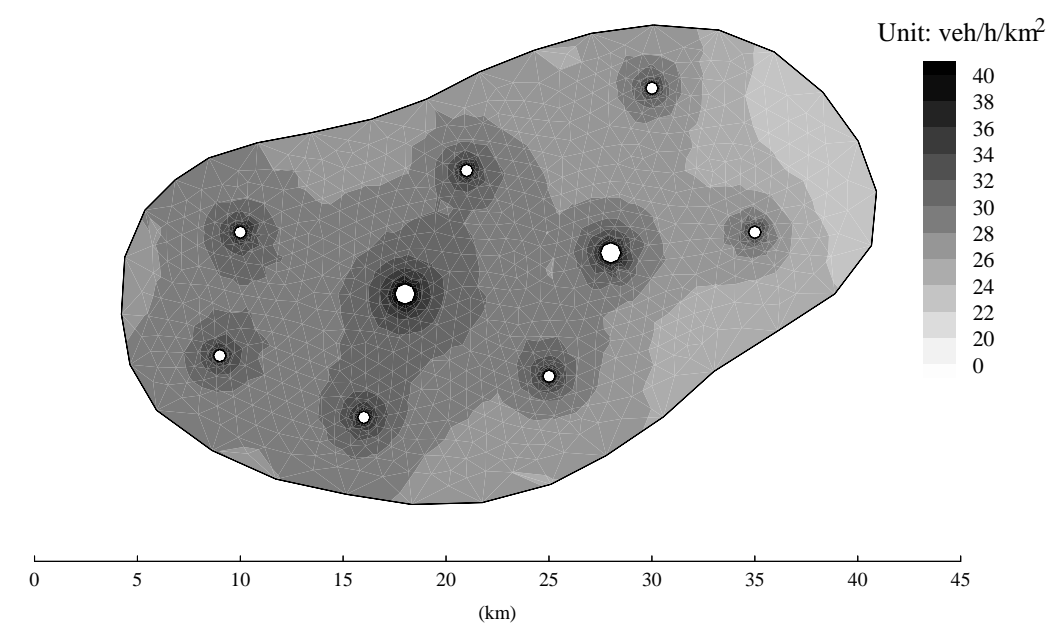

Figure 9. The travel demand to CBD 1 in the city. 


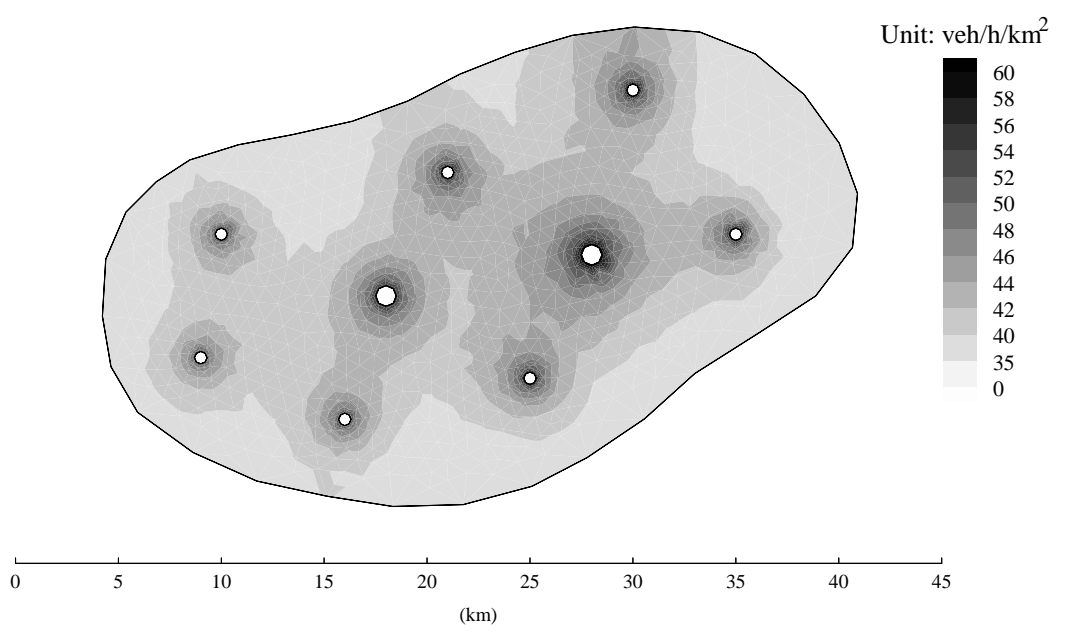

Figure 10. The travel demand to CBD 2 in the city. 